reported that, during the time under investigation, he had a thumbnail-sized wound on the medial side of the third finger of his right hand, sustained in mid-April 1998. He used a bandage for 3 or 4 days, but not thereafter, although the wound was still weeping. The assistant admitted to negligent behavior, but at the time he considered the open wound to be an old injury and was not aware that such an attitude might be risky for him and his patients. Between April 28, 1998, the day of surgery in patient 1 , and June 9,1998 , the day of surgery in patient 6 , the assistant participated in 39 operations. Between the time he went on sick leave because of acute hepatitis $\mathrm{C}$ and July 1998, another 118 operations were performed in the hospital; no further HCV infections occurred.

Numerous breaches of general infection control practices had taken place. For instance, needles were frequently recapped after use, and gloves were not always worn in settings in which exposure was likely. Multidose vials for flushing solutions, saline, local anesthetic drugs, and heparin were often used in the operating rooms, although the solutions were changed every second day. As a disinfectant for surfaces, the hospital used a product based on a peroxide compound (Dismozon pur, Bode Chemie, Hamburg, Germany) not recommended for areas grossly contaminated with blood.

This report provides evidence that a nonsurgical staff member infected with $\mathrm{HCV}$ transmitted the virus to at least five patients. The precise mechanisms leading to infection could not be determined. The only identifiable condition that might have caused the spread of the virus was the wound on the assistant's hand. Given the high plasma levels of $\mathrm{HCV}$ RNA in both patient 1 and the assistant, and given that the assistant usually did not wear gloves in the operating room, it is possible that a fraction of a microliter of blood or wound secretions might have transmitted HCV from patient 1 to the assistant and subsequently from him to the five other patients.

FROM: Ross RS, Viazov S, Gross T, Hofmann F, Seip $\mathrm{HM}$, Roggendorf $\mathrm{M}$. Transmission of hepatitis $\mathrm{C}$ virus from a patient to an anesthesiology assistant to five patients. $N$ Engl JMed 2000;343:1851-1854.

\section{Psychiatric Inpatients at Risk for HIV, TB, and Hepatitis}

In a study of 655 men and women admitted to a psychiatric hospital between 1997 and 1999, the patients were 4.5 times as likely to have hepatitis B and 11.9 times as likely to have hepatitis $\mathrm{C}$ as those in the general public. The risk seems to be increasing. In 1997 they found that $19.9 \%$ of the cohort was infected with hepatitis C; by 1999 this figure rose to $28.4 \%$. The researchers also found that $20.2 \%$ of patients tested positive for TB, which is four times the estimated rate in the general US population; the rate of HIV infection among the psychiatric patients was $2.8 \%$, nine times that seen in the general population. "It was interesting that these patients, who are directly involved in the medical system, have very poor medical care-and less than $20 \%$ have regular medical doctors that they see," lead investigator Dr. W.F. Pirl, Harvard University, Boston, Massachusetts, said. He presented the findings at the annu- al meeting of the Academy of Psychosomatic Medicine, in Palm Springs, California.

Dr. Pirl told Reuters Health he was surprised by the high infection rates in the psychiatric inpatients. "I think prevention efforts need to give more attention to substance abuse treatment," he said, adding that psychiatric inpatients are often not tested for these diseases. "Some of the things you would think would make people be tested-such as a history of drug abuse-were not indicative of whether or not they had been tested. So, in general, we need to find better ways to integrate medical doctors into the care of chronic psychiatric patients," he concluded. "All of them have some medical issues, and it would be nice to develop a system where preventative healthcare was part of mental health systems instead of being separate."

FROM: ICAN News. Nov 21, 2000.

\section{Rubella Outbreak: From Workplace to Community}

The largest outbreak of rubella in the past 5 years occurred in Nebraska in 1999. To examine risk factors for disease, susceptibility of the risk population, role of vaccine failure, and the need for new vaccination strategies, a detailed investigation was conducted of the 83 confirmed rubella cases occurring in Douglas County, Nebraska, between March 23 and August 24, 1999. Case characteristics, compared with that of the general county population; area childhood rubella vaccination rates; and susceptibility among pregnant women before versus during and after the outbreak were the main outcomes measured.

All 83 rubella cases were unvaccinated or had unknown vaccination status and fell into three groups: (1) Fifty-two (63\%) were young adults (median age, 26 years), $83 \%$ of whom were born in Latin American countries where rubella vaccination was not routine. They were either employed in meat-packing plants or were their household contacts. Attack rates in the plants were high $(14.4 / 1,000$ vs $0.19 / 1,000$ for general county population). (2) Sixteen (19\%), including 14 children ( 9 of whom were aged $<12$ months) and two parents, were US-born and non-Hispanic, who acquired the disease through contacts at two day-care facilities (attack rate, $88.1 / 1,000$ ). (3) Fifteen (18\%) were young adults (median age, 22 years) whose major disease risk was residence in population-dense census tracts where meat-packing-related cases resided ( $\mathrm{R} 2=0.343 ; P<.001) ; 87 \%$ were born in Latin America. Among pregnant women, susceptibility rates were $13 \%$ before the outbreak and $11 \%$ during and after the outbreak. Six (25\%) of 24 susceptible women tested were seropositive for rubella IgM. Rubella vaccination rates were $90.2 \%$ for preschool children and $99.8 \%$ for school-aged children.

A large rubella outbreak occurred among unvaccinated persons in a community with high immunity levels. Crowded working and living conditions facilitated transmission, but vaccine failure did not. Workplace vaccination could be considered to prevent similar outbreaks.

FROM: Danovaro-Holliday MC, LeBaron CW, Allensworth C, Raymond R, Borden G, Murray AB, et al. A 
large rubella outbreak with spread from the workplace to the community. JAMA 2000;284:2733-2739.

\section{Gene Therapy Reverses Sepsis-Induced Suppression of Lung Antibacterial Host Defense}

Sepsis syndrome frequently is complicated by the development of nosocomial infections, particularly gram-negative pneumonia. Although TNF-alpha (TNF) has been shown to mediate many of the pathophysiologic events in sepsis, this cytokine is a critical component of innate immune response within the lung. Chen and coinvestigators from University of Michigan Medical School, Ann Arbor, and Department of Microbiology, Toho University, Tokyo, Japan, hypothesized that the transient transgenic expression of TNF within the lung during the postseptic period could augment host immunity against nosocomial pathogens. To test this, mice underwent 26-gauge cecal ligation and puncture (CLP) as a model of abdominal sepsis, followed 24 hours later by intratracheal administration of Pseudomonas aeruginosa. In animals undergoing sham surgery followed by bacterial challenge, $P$ aeruginosa were almost completely cleared from the lungs within 24 hours. In contrast, mice undergoing CLP were unable to clear $P$ aeruginos $a$ and rapidly developed bacteremia.

Alveolar macrophages (AM) recovered from mice 24 hours after CLP produced significantly less TNF ex vivo, as compared with AM from sham animals. Furthermore, the adenoviral mediated transgenic expression of TNF within the lung increased survival in CLP animals challenged with $P$ aeruginosa: from $25 \%$ in animals receiving control vector to $91 \%$ in animals administered recombinant murine TNF adenoviral vector. Improved survival in recombinant murine TNF adenoviral vector-treated mice was associated with enhanced lung bacterial clearance and proinflammatory cytokine expression, as well as enhanced AM phagocytic activity and cytokine expression when cultured ex vivo. These observations suggest that intrapulmonary immunostimulation with TNF can reverse sepsis-induced impairment in antibacterial host defense.

FROM: Chen GH, Reddy RC, Newstead MW, Tateda K, Kyasapura BL, Standiford TJ. Intrapulmonary TNF gene therapy reverses sepsis-induced suppression of lung antibacterial host defense. J Immunol 2000;165:6496-6503.

\section{New Recombinant BCG Vaccines Provide Greater TB Immunity}

Horwitz and coworkers, from the School of Medicine, University of California, Los Angeles, California, have developed new recombinant bacillus Calmette-Guérin (BCG) vaccines that offer greater immunity than current commercially available vaccines. Using two commercially available strains of BCG as host strains, BCG Connaught and Tice, the investigators constructed two recombinant $\mathrm{BCG}$ vaccines stably expressing and secreting the $30 \mathrm{kDa}$ major secretory protein of Mycobacterium tuberculosis. They tested the efficacy of the two strains in the highly susceptible guinea pig model of pulmonary TB, a model noteworthy for its close resemblance to human TB.

Animals immunized with the recombinant $B C G$ vaccines and challenged by aerosol with a highly virulent strain of $M$ tuberculosis had 0.5 logs fewer $M$ tuberculosis in their lungs and $1 \log$ fewer bacilli in their spleens on average than animals immunized with their parental conventional BCG vaccine counterparts. Statistically, these differences were highly significant. Paralleling these results, at necropsy, animals immunized with the recombinant BCG vaccines had fewer and smaller lesions in the lung, spleen, and liver and significantly less lung pathology than animals immunized with the parental $B C G$ vaccines.

The recombinant vaccines are the first vaccines against TB more potent than the current commercially available BCG vaccines, which were developed nearly a century ago.

FROM: Horwitz MA, Harth G, Dillon BJ, MaslesaGalic. Recombinant bacillus Calmette-Guérin ( $\mathrm{BCG}$ ) vaccines expressing the Mycobacterium tuberculosis $30-\mathrm{kDa}$ major secretory protein induce greater protective immunity against tuberculosis than conventional BCG vaccines in a highly susceptible animal model. Proc Natl Acad Sci USA 2000;97:13853-13858.

\section{Increase in TB Among Foreign-Born Persons in US, 1993-1998}

Immigration is a major force sustaining the incidence of TB in the United States. Data from case reports on TB were used to describe trends and characteristics of foreign-born persons with TB and the implications for TB program planning and policy development.

The number of TB cases among foreign-born persons increased $2.6 \%$, from 7,402 in 1993 to 7,591 in 1998; the proportion of US cases that were foreign-born increased from $29.8 \%$ to $41.6 \%$. During 1993 to 1998 , the TB case rate was 32.9 per 100,000 population in foreign-born persons, compared with 5.8 per 100,000 in US-born persons. Six states reported $73.4 \%$ of foreign-born cases (California, New York, Texas, Florida, New Jersey, and Illinois). Approximately two thirds of these cases were originally from Mexico, the Philippines, Vietnam, India, China, Haiti, and South Korea. Among those for whom date of US entry was known, $51.5 \%$ arrived 5 years or less prior to the diagnosis of TB. Most were male and aged 25 to 44 years. During 1993 to 1996 , the proportion receiving some portion of treatment under directly observed therapy increased from $27.3 \%$ to $59.1 \%$; approximately $70 \%$ completed therapy in 12 months. The rate of primary resistance to isoniazid was $11.6 \%$ and to both isoniazid and rifampin was $1.7 \%$.

As the United States moves toward the goal of TB elimination, success will depend increasingly on reducing the impact of TB in foreign-born persons. Continued efforts to tailor local TB control strategies to the foreign-born community and commitment to the global TB battle are essential.

FROM: Talbot EA, Moore M, McCray E, Binkin NJ. Tuberculosis among foreign-born persons in the United States, 1993-1998. JAMA 2000;284:2894-2900. 